\title{
ADUBAÇÃO POTÁSSICA NA PRODUTIVIDADE DA SOQUEIRA DE CANA-DE-AÇÚCAR COLHIDA SEM QUEIMA
}

\author{
FELTRIN, Mariana Sanches ${ }^{1}$ \\ LAVANHOLI, Maria das Graças Drumsta Prado ${ }^{2}$ \\ SILVA, Helena Sanches ${ }^{3}$ \\ PRADO, Renato de Mello ${ }^{4}$
}

Recebido em: 2009.12.24

Aprovado em: 2010.02 .22

ISSUE DOI: $10.3738 / 1982.2278-334$

RESUMO: O objetivo desse trabalho foi de verificar o efeito da aplicação do potássio na produtividade da soqueira de cana-de-açúcar de primeiro corte colhida sem despalha à fogo. $\mathrm{O}$ experimento foi instalado na fazenda Nova Aliança em Sales de Oliveira-SP, Brasil, na primeira soqueira de cana-de-açúcar (SP 80-1816). O experimento foi avaliado em delineamento inteiramente casualizado com 5 tratamentos e 4 repetições. Os tratamentos foram constituídos por doses de potássio da seguinte forma: 1) 0 e $60 \mathrm{~kg} \mathrm{ha}^{-1} \mathrm{~K}_{2} \mathrm{O}$; 2) 0 e $90 \mathrm{~kg} \mathrm{ha}^{-1}$ $\mathrm{K}_{2} \mathrm{O}$; 3) 0 e $120 \mathrm{~kg} \mathrm{ha}^{-1} \mathrm{~K}_{2} \mathrm{O}$; 4) 60 e $60 \mathrm{~kg} \mathrm{ha}^{-1} \mathrm{~K}_{2} \mathrm{O}$; 5) 60 e $120 \mathrm{~kg} \mathrm{ha}^{-1} \mathrm{~K}_{2} \mathrm{O}$, aplicados aos 30 e 60 dias após a brotação da soqueira respectivamente. Avaliaram-se os parâmetros tecnológicos (pH, pol\%cana, pureza, fibra, açúcares redutores e açúcares totais recuperáveis) e a produção de colmos e de açúcar. A aplicação do potássio em doses maiores que $60 \mathrm{~kg} \mathrm{ha}^{-1} \mathrm{~K}_{2} \mathrm{O}$ não afetou a qualidade tecnológica da soqueira de cana-de-açúcar, entretanto a dose de $120 \mathrm{~kg} \mathrm{ha}^{-1} \mathrm{~K}_{2} \mathrm{O}$ dividida em dois parcelamentos proporcionou maior produção de colmos. Concluiu-se que o incremento das doses de potássio é mais importante na produção de colmos do que para a qualidade tecnológica da soqueira de cana-de-açúcar.

Palavras-chave: Potássio. Adubação. Saccharum spp.

SUMMARY: An experiment was performed where the aim was to appraise the response of the potassium fertilization allotment in root reaped sugarcane. The experiment was installed on the Nova Aliança farm in Sales de Oliveira, São Paulo, Brazil, on the first root sugarcane (SP 80-1816). The experiment was appraised in delineation entirely casually witch 5 treatments and 4 repetitions. The treatments were constituted by rate of potassium as following: 1) 0 and $60 \mathrm{~kg} \mathrm{ha}^{-1} \mathrm{~K}_{2} \mathrm{O}$;2) 0 and $90 \mathrm{~kg} \mathrm{ha}^{-1} \mathrm{~K}_{2} \mathrm{O}$; 3) 0 and $120 \mathrm{~kg} \mathrm{ha}^{-1} \mathrm{~K}_{2} \mathrm{O}$; 4) 60 and 60 $\mathrm{kg} \mathrm{ha}^{-1} \mathrm{~K}_{2} \mathrm{O}$; 5) 60 and $120 \mathrm{~kg} \mathrm{ha}^{-1} \mathrm{~K}_{2} \mathrm{O}$, applied to 30 and 60 days after the root sprout respectively. Technological parameters were appraised $(\mathrm{pH}$, pol\% sugarcane, purity, fiber, redactor sugar and recoverable total sugar) and the culms and sugar production. The potassium application in higher doses than $60 \mathrm{~kg} \mathrm{ha}^{-1} \mathrm{~K}_{2} \mathrm{O}$ didn't affect the technological quality of the root sugarcane, in the meantime the rate of $120 \mathrm{~kg} \mathrm{ha}^{-1} \mathrm{~K}_{2} \mathrm{O}$ divided into two allotments made the culms production higher. It was concluded that the rate of potassium increment is more important in the culms production than for the root sugarcane technological quality.

Keywords: potassium, fertilization, Saccharum spp.

\section{INTRODUÇÃO}

Nos próximos 10 anos, a produção brasileira de cana-de-açúcar deverá crescer cerca de 50\%, atingindo 557 milhões de toneladas na safra 2013/14. A produção deverá continuar

\footnotetext{
${ }^{1}$ Acadêmica da Faculdade Dr. Francisco Maeda - Ituverava, SP..

${ }^{2}$ Prof $^{a}$. Dra ${ }^{a}$ da Faculdade Dr. Francisco Maeda - Ituverava, SP.

${ }^{3}$ Eng $^{a}$. Agr ${ }^{\mathrm{a}}$. Usina Vale do Rosário Agrícola - São Joaquim da Barra, SP

${ }^{4}$ Prof. Dr. Faculdade de Ciências Agrárias e Veterinárias - UNESP, Jaboticabal, SP.
} 
concentrada no centro-sul, pois o Brasil apresenta alto consumo de álcool carburante se comparado aos demais países. Por essa razão e pelo fato de que o país já está sendo autosuficiente em petróleo, acredita-se que haverá maiores estímulos para exportação do álcool (NEHMI FILHO, 2005).

Pelo fato dessa grande expansão do mercado sucroalcooleiro no Brasil, os estudos sobre a cultura da cana-de-açúcar têm-se intensificado cada vez mais, com o objetivo de aumentar a potencialidade das variedades cada vez mais produtivas, com uso de técnicas novas de manejo, defesa da planta e com o atendimento das exigências nutricionais da planta, especialmente em solos tropicais que caracterizam pela baixa fertilidade.

É conhecido que os solos tropicais apresentam baixo teor de potássio e como esse nutriente é um dos mais absorvidos pela cana-de-açúcar especialmente na fase vegetativa (RAIJ, 1991), sendo importante na nutrição das plantas, pois desempenha várias funções como: regulação da turgidez do tecido, ativação enzimática, abertura e fechamento dos estômatos; transporte de carboidratos, transpiração, resistência geada, seca, doenças e ao acamamento (MALAVOLTA, 1980), nota-se a importância deste macronutriente na produtividade das culturas, como da cana-de-açúcar.

Em cana-de-açúcar a deficiência em potássio induz a um crescimento reduzido, os colmos se tornam finos, os internódios ficam curtos, diminui a brotação entre outros. E observa-se também que o sintoma mais típico de deficiência é o aparecimento de manchas avermelhadas nas folhas (HAAG; ACCORSI, 1978).

Os estudos sobre a adubação potássica para a cana-de-açúcar são necessários devido a mudança no sistema de cultivo dessa planta, que era colheita com despalha a fogo, e atualmente seria sem uso do fogo, tendo a presença da palhada pelo fato do corte mecanizado. Nesse novo sistema de cultivo, os estudos sobre adubação estão concentrados para o nitrogênio, devido aos fenômenos de perdas, especialmente por volatilização (COSTA et al, 2003; VITTI et al, 2007). E a presença da palhada na superfície do solo pode liberar o potássio para o solo fornecendo o nutriente para soqueira de cana-de-açúcar (ROSSETTO; DIAS, 2005), beneficiando a nutrição da plantas e podendo diminuir a necessidade de adubação deste nutriente em sistema de colheita sem despalha a fogo, comparado ao sistema com uso do fogo.

Os resultados da literatura com relação ao potássio indicam efeitos positivos na produção de cana-de-açúcar com despalha à fogo (SAMPAIO, 1945; ESPIRONELO et al. 1997; LANA et al. 2004; ROSSETTO et al. 2004), entretanto, outros trabalhos não indicam efeito na produção de cana-de-açúcar ( ALVAREZ et al. 1960; ALVAREZ; PACHECO, 
1962). Diante dessa situação há uma necessidade de realizar mais trabalhos sobre os efeitos do potássio na cana-de-açúcar e especialmente para as soqueiras cultivada sem o uso da despalha à fogo.

O objetivo desse trabalho foi de verificar o efeito da aplicação do potássio na produtividade e na qualidade de cana-de-açúcar em soqueira de primeiro corte colhida sem despalha à fogo.

\section{MATERIAL E MÉTODOS}

O experimento foi conduzido na fazenda Nova Aliança, no município de Sales de Oliveira-SP. Foi utilizada uma área com soqueira de primeiro corte, em um Latossolo Vermelho distrófico, tendo as seguintes características (camada de 0-40 $\mathrm{cm}$ de profundidade na entrelinha da cultura): $\mathrm{pH}$ em $\mathrm{CaCl}_{2}$ : 5,7; M.O.(\%): 34; P: $24 \mathrm{mmol}_{\mathrm{c}} \mathrm{dm}^{-3}$; K: 0,8 $\mathrm{mmol}_{\mathrm{c}} \mathrm{dm}$ ${ }^{-3}$; Ca: 27 mmol $_{\mathrm{c}} \mathrm{dm}^{-3}$; $\mathrm{Mg}: 8 \mathrm{mmol}_{\mathrm{c}} \mathrm{dm}^{-3}$; $\mathrm{H}+\mathrm{Al}: 21 \mathrm{mmol}_{\mathrm{c}} \mathrm{dm}^{-3}$; SB: $36 \mathrm{mmol}_{\mathrm{c}} \mathrm{dm}^{-3}$; CTC:

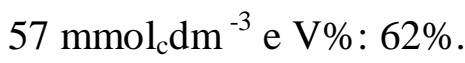

A variedade de cana-de-açúcar utilizada foi a SP 80-1816 que apresenta ótima brotação de soca, média exigência em fertilidade do solo, bom perfilhamento, maturação média, teor de sacarose elevado, alto teor de fibra, e não apresenta florescimento (SORDI et al. 2003).

A parcela constitui-se de $70 \mathrm{~m}^{2}$, ou seja, cinco linhas de dez metros de comprimento, tendo 1,5 m nas laterais como bordaduras.

O experimento foi constituído por cinco tratamentos (TABELA 1), utilizando como fonte de potássio, o cloreto de potássio $\left(60 \%\right.$ de $\left.\mathrm{K}_{2} \mathrm{O}\right)$, aplicado em dois parcelamentos 30 e 90 dias após a brotação da primeira soqueira.

TABELA 1: Tratamentos utilizados no experimento de potássio de primeira soqueira de cana-de-açúcar.

\begin{tabular}{lcc}
\hline Tratamentos & $\mathbf{1}^{\circ}$ Parcelamento & $\mathbf{2}^{\circ}$ Parcelamento \\
\hline & 0 & 60 \\
$\mathbf{2}$ & 0 & $\mathbf{k g ~ h a}^{1} \mathbf{K}_{\mathbf{2}} \mathbf{O}-$ \\
$\mathbf{3}$ & 0 & 90 \\
$\mathbf{4}$ & 60 & 120 \\
$\mathbf{5}$ & 60 & 60 \\
\hline
\end{tabular}


O fertilizante potássico foi aplicado na superfície a $30 \mathrm{~cm}$ da linha da cana-de-açúcar sobre a palhada, sem incorporação. Além do potássio, aplicou-se em cobertura junto com o primeiro parcelamento (30 dias) $102 \mathrm{~kg} \mathrm{ha}^{-1}$ de $\mathrm{N}$ na forma de nitrato de amônio (34\% de $\mathrm{N})$.

Durante o período experimental, avaliou-se a temperatura máxima e mínima mensal e também a precipitação mensal (TABELA 2). Nota-se que não houve estresse hídrico importante durante o desenvolvimento da cultura.

O plantio da cana-de-açúcar foi realizado de modo convencional, com sulcos na profundidade de $20 \mathrm{~cm}$, espaçamento entre sulcos de $150 \mathrm{~cm}$ e mudas na quantidade de duas canas cruzadas (pé com ponta). Após a colheita da cana-planta, no dia 29 de setembro de 2004, instalou-se o experimento na área.

$\mathrm{Na}$ área experimental não foram realizadas aplicação de vinhaça e de torta de filtro. $\mathrm{O}$ controle de plantas daninhas do experimento utilizado foi o químico com aplicação de 3,6 L $\mathrm{ha}^{-1}$ de Amitrina, 2,6 $\mathrm{L} \mathrm{ha}^{-1}$ de MSMA e 0,3 $\mathrm{L} \mathrm{ha}^{-1}$ de Adjuvante.

Aos 12 meses após a brotação da soqueira, no dia 20 de outubro de 2005, realizou-se a colheita, obtendo-se a produção de colmos. Em cada parcela coletaram-se dez colmos em seqüência, aleatoriamente na parcela para análise tecnológica. Assim avaliou-se no caldo o $\mathrm{pH}$, Pol (teor de sacarose) e na cana, a massa do bolo de bagaço úmido (PBU), para se calcularem PC (\% em massa da sacarose), fibra, AR (açúcar redutor do caldo) e ATR (açúcar teórico recuperável) (Fernandes, 2003). E ainda realizou-se o cálculo da produção de açúcar com base na produção de colmos e de ATR.

TABELA 2 - Temperatura (máxima e mínima) e precipitação mensal ao longo do período experimental - Fazenda Nova Aliança (2004 e 2005).

(Continua)

\begin{tabular}{lcccccc}
\hline \multirow{2}{*}{ Mês/Ano } & \multicolumn{3}{c}{$\mathbf{2 0 0 4}$} & \multicolumn{3}{c}{$\mathbf{2 0 0 5}$} \\
\cline { 2 - 6 } & $\mathbf{T}^{\circ}$ Máx. & $\mathbf{T}^{\circ}$ Min. & Precipitação & $\mathbf{T}^{\circ}$ Máx. & $\mathbf{T}^{\circ}$ Min. & Precipitação \\
\hline \multirow{2}{*}{ Janeiro } & 30,9 & 19,8 & 266,0 & 31,6 & 20,5 & mm \\
Fevereiro & 29,6 & 18,3 & 347,0 & 32,3 & 18,6 & 106,0 \\
Março & 30,9 & 30,3 & 47,4 & 31,2 & 19,3 & 118,2 \\
Abril & 27,6 & 13,2 & 190,5 & 31,6 & 18,1 & 27,7 \\
Maio & 27,3 & 13,2 & 93,4 & 29,5 & 13,6 & 164,3 \\
Junho & 27,3 & 11,7 & 14,7 & 28,1 & 13,2 & 35,1 \\
Julho & 27,5 & 11,4 & 20,0 & 26,8 & 11,7 & 25,9 \\
Agosto & 30,8 & 11,6 & 0,0 & 31,1 & 13,1 & 11,0 \\
\hline
\end{tabular}


TABELA 2 - Temperatura (máxima e mínima) e precipitação mensal ao longo do período experimental - Fazenda Nova Aliança (2004 e 2005).

(Conclusão)

\begin{tabular}{lcccccc}
\hline Setembro & 34,5 & 14,9 & 1,6 & 30,2 & 16,9 & 77,6 \\
Outubro & 31,2 & 16,0 & 127,2 & 33,8 & 20,0 & 95,3 \\
Novembro & 31,2 & 18,1 & 198,3 & 30,8 & 18,7 & 143,6 \\
Dezembro & 30,9 & 15,7 & 288,6 & 30,6 & 16,8 & 239,6 \\
\hline Média & 30,2 & 15,7 & - & 30,6 & 16,8 & - \\
Acumulado & - & - & 1595,0 & - & - & 1336,3 \\
\hline
\end{tabular}

O delineamento utilizado foi inteiramente casualizado, com 4 repetições com base nos resultados obtidos realizou-se a análise de variância para as diversas variáveis estudadas e utilizou-se o teste de comparação de médias Duncan a 5\% de probabilidade.

\section{RESULTADOS E DISCUSSÃO}

Não se observaram diferenças significativas entre os tratamentos para os diferentes variáveis tecnológicos analisados (TABELA 3).

TABELA 3 - Médias da análise tecnológica da cana-de-açúcar em função dos tratamentos utilizados.

\begin{tabular}{lccccc}
\hline Tratamentos & $\mathbf{p H}$ & $\mathbf{P C}^{\mathbf{1}}$ & Fibra & $\mathbf{A T R}^{\mathbf{2}}$ & $\mathbf{A R}^{\mathbf{3}}$ \\
\hline & & & & & \\
1 & 5,07 & 17,91 & 12,58 & 169,02 & 0,35 \\
2 & 5,03 & 17,84 & 13,25 & 168,34 & 0,33 \\
3 & 5,03 & 17,77 & 13,43 & 167,63 & 0,33 \\
4 & 5,05 & 17,54 & 12,94 & 165,65 & 0,35 \\
5 & 5,09 & 18,12 & 13,20 & 170,85 & 0,30 \\
\hline Média geral & 5,05 & 17,84 & 13,08 & 168,30 & 0,33 \\
Teste F & $0,83^{\mathrm{NS}}$ & $0,42^{\mathrm{NS}}$ & $0,58^{\mathrm{NS}}$ & $0,42^{\mathrm{NS}}$ & $0,60^{\mathrm{NS}}$ \\
C.V. $(\%)$ & 1,2 & 3,7 & 6,7 & 3,5 & 3,7 \\
${ }^{\top}$ PC: pol\% da cana; ${ }^{2}$ ATR: açúcar teórico recuperável; ${ }^{3}$ AR: açúcar recuperável. &
\end{tabular}

Os teores médios de PC elevados são decorrentes da época de colheita, doze meses após o plantio, ou seja, com cana completamente madura. Os teores de fibra (média de 13,08) são consistentes com as características da variedade. Os demais parâmetros estão de 
acordo com a fase do ciclo e com as características varietais (SORDI et al 2003).

Pelos resultados obtidos, a aplicação de $60 \mathrm{~kg} \mathrm{ha}^{-1} \mathrm{~K}_{2} \mathrm{O}$ em cobertura na soqueira sem o parcelamento é suficiente para manter a qualidade tecnológica da cana-de-açúcar. Esses resultados discordam do trabalho realizado por Lana et al. (2004) que observaram efeito positivo do parcelamento do potássio para cana-planta. Essa diferença possivelmente deve-se ao ciclo da cultura, onde na cana-planta tem-se efeito favorável do parcelamento do potássio e nas soqueiras esse efeito não ocorre. Na cana-planta, a adubação potássica no sulco, pode além de causar danos à brotação das gemas devido à alta salinidade do cloreto de potássio, também pode ser facilmente perdido por lixiviação.

Estes resultados sobre a ausência de efeito do potássio na qualidade da cana, discordam de Hauck; Dickinson (1954) e de Sampaio (1945), que observou que a adubação potássica favorece a formação do açúcar do caldo, não se notando o mesmo efeito na melhoria da pureza. Entretanto, os resultados obtidos estão de acordo com o trabalho realizado por Alvarez et al. (1960) e por Alvarez e Pacheco (1962) que não verificaram efeito do potássio na qualidade tecnológica da cana-de-açúcar.

Notou-se que o aumento da dose de potássio na soqueira de cana-de-açúcar da dose de $60 \mathrm{~kg} \mathrm{ha}^{-1} \mathrm{~K}_{2} \mathrm{O}$ (Tratamento 1) para a dose de $120 \mathrm{~kg} \mathrm{ha}^{-1} \mathrm{~K}_{2} \mathrm{O}$ parcelada (tratamento 4), resultou em maior produção de colmos (TABELA 4). Os resultados obtidos concordam com a revisão de Rossetto et al. (2004) sobre ensaios de adubação com potássio realizados no estado de SP, e concluíram que para esse macronutriente, a resposta da cana foi de natureza linear em sete das dez avaliações realizadas, tanto em cana-planta como em soqueiras.

TABELA 4 - Média das análises dos parâmetros de produção de colmos e de açúcar em função dos tratamentos utilizados.

\begin{tabular}{lcc}
\hline Tratamento & Produção de colmos & Produção de açúcar \\
\hline $\mathbf{1}$ & $93,31 \mathrm{~b}$ & 16,18 \\
$\mathbf{2}$ & $95,29 \mathrm{~b}$ & 16,00 \\
$\mathbf{3}$ & $97,59 \mathrm{~b}$ & 15,39 \\
$\mathbf{4}$ & $103,23 \mathrm{a}$ & 17,06 \\
$\mathbf{5}$ & $100,26 \mathrm{~b}$ & 17,15 \\
\hline Média geral & 98,54 & 16,36 \\
Teste F & $3,7 *$ & $1,28^{\mathrm{NS}}$ \\
C.V.(\%) & 8,4 & 8,0 \\
\hline
\end{tabular}

Letras iguais não diferem entre si pelo teste de Duncan 5\% de probabilidade. 
Observou-se que o tratamento $4\left(60\right.$ e $\left.60 \mathrm{~kg} \mathrm{ha}^{-1} \mathrm{~K}_{2} \mathrm{O}\right)$ proporcionou a maior produção de colmos e portanto, o emprego de maior dose de potássio sem o primeiro parcelamento (120 $\mathrm{kg} \mathrm{ha}^{-1} \mathrm{~K}_{2} \mathrm{O}$ ) (tratamento 3), não esteve associado com maior produção de colmos (TABELA 4). Assim esses resultados discordam de Spironello et al. (1997), que indicava dose de potássio de $130 \mathrm{~kg} \mathrm{ha}^{-1} \mathrm{~K}_{2} \mathrm{O}$ para soqueiras em condições de solo e de produtividade próxima do presente experimento.

Por fim, notou-se que a adubação potássica apesar de ter afetado positivamente a produção de colmos (TABELA 4) não afetou a qualidade da cana (TABELA 3). Entretanto, Alvarez e Pacheco (1962) verificaram que a aplicação de $100 \mathrm{~kg} \mathrm{ha}^{-1} \mathrm{~K}_{2} \mathrm{O}$ proporcionou aumento de $10 \mathrm{t} \mathrm{ha}^{-1}$ de cana-de-açúcar e também de $1,34 \mathrm{t} \mathrm{ha}^{-1}$ de açúcar comparado com a testemunha, o que é explicado pelo incremento dos colmos por área. Assim, o presente trabalho indica que o potássio não tem efeito nas qualidades tecnológicas (pol) por unidade de colmo, suficiente para incrementar a produção de açúcar por área.

\section{CONCLUSÃO}

A aplicação do potássio em doses maiores que $60 \mathrm{~kg} \mathrm{ha}^{-1} \mathrm{~K}_{2} \mathrm{O}$ não afetou a qualidade tecnológica da soqueira de cana-de-açúcar, entretanto, a dose de $120 \mathrm{~kg} \mathrm{ha}^{-1} \mathrm{~K}_{2} \mathrm{O}$ dividida em dois parcelamentos proporcionou maior produção de colmos. $\mathrm{O}$ incremento das doses de potássio é mais importante na produção de colmos do que para a qualidade tecnológica da soqueira de cana-de-açúcar.

\section{AGRADECIMENTOS}

Á Prof ${ }^{a}$. Dr ${ }^{\mathrm{a}}$. Maria das Graças Drumsta Lavanholi, na orientação desse trabalho da primeira autora. Ao grupo Vale do Rosário-Agrícola, em especial a Helena Sanches Silva Renato Ferreira da Rosa pela oportunidade de realização do trabalho.

\section{REFERÊNCIAS}

ALVAREZ, R.; PACHECO, C.A.J. Adubação da cana-de-açúcar; VII - Ensaio preliminar de adubação N-P-K no arenito de Bauru, Bragantia, v.22, n.17, 1963.

ALVAREZ, R.; AMARAL, Z.A.; ARRUDA, V.H. Ensaio de adubação N-P-K em cana-deaçúcar. Bragantia, v.19, n.63,1960.

COSTA, M.C.G., VITTI, G.C.; CANTARELLA, H. N-NH $\mathrm{N}_{3}$ losses from nitrogen sources applied over unburned sugarcane straw. Revista Brasileira de Ciência do Solo, v.27, n.4,p.631-637, 2003. 
FERNANDES, A.C. Cálculos na agroindústria de cana-de-açúcar. 2 ed. Piracicaba: STAB Sociedade dos Técnicos Açucareiros e Alcooleiros do Brasil, p.18 -20, 2003.

HAUCK, F.W.; DICKINSON, J. Conveniência y experimentacion con potasio en el cultivo de caña de azucar. ATAC - R. Técnica Inf.,v.13,p.626-635, 1954.

HAAG, H.P.; ACCORSI, W.R. Deficiência de macro e micronutriente em cana-de-açúcar variedade CB 41-76 cultivada em solução nutritiva. Anais da ESALQ, v.35, 1978.

LANA, R.M.Q. et al. Parcelamento da adubação potássica na cana-planta. Revista STAB Açúcar, Álcool e Subprodutos, v. 23, n. 2, p. 28-31, 2004.

MALAVOLTA, E. Elementos de nutrição de plantas. São Paulo: Agronômica Ceres, 251p, 1980.

NEHMI FILHO, V.A. Alta do Petróleo e Vitória na OMC reforçam expectativas altistas. Cana-de-açúcar. São Paulo: FNP, Mercado T. Perspectivas, p.261-264, 2005.

RAIJ, V.B. Fertilidade do solo e adubação. Piracicaba: Ceres,1991. p. 117-136

ROSSETTO R.; DIAS, F.L.F. Nutrição e adubação da cana-de-açúcar: indagações e reflexões. POTAFOS, 2005. (Encarte técnico - Informações Agronômicas n 110).

ROSSETO, R.; SPIRONELLO, A.; CANTARELLA, H. Calagem para cana-de-açúcar e sua interação com a adubação potássica. Bragantia, v. 63, n.1, p. 105-119, 2004.

SORDI, A. R. Caracterização morfológica e agroindustrial de variedades de cana-deaçúcar. Centro de Tecnologia Copersucar, Coordenadoria de Variedades, Programa de Caracterização de Clones. 2003. (Boletim).

SAMPAIO, S.C. A composição do caldo de cana-de-açúcar: contribuição para o estudo da adubação. Bragantia,v.5, n.5. p.291-308, 1945.

SPIRONELLO, A.et al. Outras culturas industriais. In: RAIJ, B.van et al. Recomendações de adubação e calagem para o estado de São Paulo. 2 ed. Campinas: Instituto Agronômico, p.237-239. 1997. (Boletim técnico, 100).

VITTI, A.C.et al. Produtividade da cana-de-açúcar relacionada à localização de adubos nitrogenados aplicados sobre os resíduos culturais em canavial sem queima. Revista Brasileira de Ciência do Solo,v. 31, n.3,p.491-498,2007. 